\title{
A colorectal cancer prediction model using traditional and genetic risk scores in Koreans
}

\author{
Keum Ji Jung ${ }^{1}$, Daeyoun Won², Christina Jeon ${ }^{3}$, Soriul Kim', Tae II Kim', Sun Ha Jee ${ }^{3 *}$ and Terri H Beaty ${ }^{5}$
}

\begin{abstract}
Background: Genome-wide association studies have identified numerous single nucleotide polymorphisms (SNPs) as associated with colorectal cancer (CRC) risk in populations of European descent. However, their utility for predicting risk to CRC in Asians remains unknown. A case-cohort study (random sub-cohort $N=1,685$ ) from the Korean Cancer Prevention Study-II (KCPS-II) $(N=145,842)$ was used. Twenty-three SNPs identified in previous 47 studies were genotyped on the KCPS-II sub-cohort members. A genetic risk score (GRS) was calculated by summing the number of risk alleles over all SNPs. Prediction models with or without GRS were evaluated in terms of the area under the receiver operating characteristic curve (AUROC) and the continuous net reclassification index (NRI).

Results: Seven of 23 SNPs showed significant association with CRC and rectal cancer in Koreans, but not with colon cancer alone. AUROCs (95\% Cl) for traditional risk score (TRS) alone and TRS plus GRS were $0.73(0.69-0.78)$ and 0.74 (0.70-0.78) for CRC, and $0.71(0.65-0.77)$ and $0.74(0.68-0.79)$ for rectal cancer, respectively. The NRI (95\% Cl) for a prediction model with GRS compared to the model with TRS alone was $0.17(-0.05-0.37)$ for CRC and 0.41 (0.10-0.68) for rectal cancer alone.

Conclusion: Our results indicate genetic variants may be useful for predicting risk to CRC in the Koreans, especially risk for rectal cancer alone. Moreover, this study suggests effective prediction models for colon and rectal cancer should be developed separately.
\end{abstract}

Keywords: Single nucleotide polymorphisms, Gene-traditional risk score, Colorectal cancer

\section{Background}

According to the Korean National Cancer Center, the incidence of colorectal cancer (CRC), the $3^{\text {rd }}$ most common cancer in Korea, has increased from 21.2/100,000 people in 1999 to 39.0/100,000 people in 2011 [1]. Steady increases in the incidence of CRC should be expected, partly due to environmental factors such as increased Western dietary patterns. Early discovery of high-risk groups could be helpful in managing risk factors and ultimately in reducing CRC incidence and mortality [2].

Previous studies have proposed CRC prediction models but these attained only limited predictive power [3,4]. Some models reflect only one aspect of the associated risk factors and failed to incorporate both the genetic and traditional risk factors (including environmental factors)

\footnotetext{
* Correspondence: jsunha@yuhs.ac

${ }^{3}$ Institute for Health Promotion and Department of Epidemiology and Health Promotion, Graduate School of Public Health, Yonsei University, 50 Yonse-ro, Seodaemun-gu, Seoul, South Korea

Full list of author information is available at the end of the article
}

of CRC [3-5]. Moreover, many previous models did not distinguish between the colon and rectal cancer, which are distinct by anatomic sites and other characteristics $[2,6]$. In fact, previous publications have reported colon and rectal cancer show different associations with traditional risk factors [7-9]. Therefore, to develop more effective prediction models, we should 1) include information on both genetic and traditional risk factors, and 2) distinguish between colon and rectal cancers.

For our CRC predictive model, the most appropriate traditional risk factors were determined from a prospective cohort study of the general Korean population. Also, after incorporating genetic factors into the model, its utility was carefully evaluated. Our study provides evidence that considering genetic factors as well as traditional risk factors in risk prediction models can improve their utility. 


\section{Results}

We attained 633,210 person-years (PY) after following 145,842 study subjects through December 2012. During the follow-up period, 258 CRC patients were verified from the National Cancer Center cancer registry database. Overall incidence rate per 100,000 PY was 40.7.

Table 1 shows the characteristics of all study participants. Participants from the KCPS-II cohort and sub-cohort had similar characteristics of age, sex, BMI, smoking status, alcohol drinking, exercise, and family history. In each cohort, the case group was older and had higher BMI and fasting blood glucose than did the control group. Also, in each cohort, the patient group showed higher rates of smoking and more cases reported a family history of CRC.

Table 2 shows the estimated hazards ratio (HR) of various factors contributing to the risk of CRC. Each cohort showed similar findings between participants in the whole KCPS-II cohort and the sub-cohort participants. Age, sex, fasting serum glucose, smoking status, exercise, and family history were ultimately selected as predictors for CRC.

Table 3 shows allelic association with CRC, colon, and rectal cancer, respectively. Depending on the cancer location (colon or rectum), each SNP showed a different pattern of association. A total of 5 out of 23 SNPs showed significant association only with rectal cancer, but not on colon cancer. A total of 2 out of 23 SNPs showed a positive association across both colon and rectum cancer, although it was only moderately significant.

In this study, the GRS was based on 7 SNPs (rs3802842, rs4939827, rs6983267, rs10505477, rs10795668, rs961253, and rs9929218). Overall these GRS followed a normal distribution (data not shown).
Table 4 shows the predictive power of models incorporating GRS with TRS for CRC, and rectal cancer using both the ROC area and NRI. AUROC (95\% CI) for TRS alone was 0.73 (0.69-0.78) for CRC, and 0.71 (0.65-0.77) for rectal cancer alone. The AUROC (95\% CI) for the combined model with both TRS and GRS was increased, especially for rectal cancer [0.74 (0.68-0.79)]. NRI (95\% CI) for the model with GRS compared to the model with only TRS was $0.17(-0.05-0.37)$ for CRC, and 0.41 $(0.10-0.68)$ for rectal cancer. Table 4 also shows the risk of CRC and rectal cancer alone after dividing GRS into quartiles. Compared with participants in the lowest quartile, those with the highest quartile of GRS had a 2.65-fold higher risk for CRC and a 10.83-fold higher risk for rectal cancer alone, respectively.

Figure 1 shows the combined risk of CRC and rectal cancer separately after dividing each GRS and TRS into quartiles. As the GRS increased into quartile 4 (Q4), the $\mathrm{CRC}$ risk increased. Also, as the TRS increased in quartile 4 (Q4), the CRC risk increased even more. Participants with TRS and GRS in the highest quartile (Q4) were determined to have about 25 times higher risk of CRC than those with TRS and GRS in the lowest quartile (Q1). Likewise, participants with TRS and GRS in the highest quartile $(\mathrm{Q} 4)$ were determined to have about 40 times as much risk of rectal cancer compared to those with TRS and GRS in the lowest quartile (Q1).

\section{Discussion}

Gene-based prediction of CRC in literatures

The heritability of risk to CRC is estimated to be $\sim 35 \%$ [10] but only about $5 \%$ of CRC cases can be attributable to highly penetrant mutations in recognized genes.

Table 1 General characteristics of study participants: The Korean Cancer Prevention Study-II and the KCPS-II sub-cohort

\begin{tabular}{|c|c|c|c|c|c|c|c|}
\hline & & \multicolumn{3}{|c|}{ KCPS-II cohort (Whole participants) } & \multicolumn{3}{|c|}{ KCPS-II sub-cohort (Case-cohort design) } \\
\hline & & CRC & No CRC & $P$ & CRC & No CRC & $P$ \\
\hline $\mathrm{N}$ & & 258 & 145,584 & & 173 & 1,514 & \\
\hline Age, year & & $50.7 \pm 10.5$ & $41.1 \pm 10.3$ & $<0.001$ & $49.7 \pm 10.9$ & $40.1 \pm 9.4$ & $<0.001$ \\
\hline Sex, $\%$ of female & & 24.1 & 37.9 & $<0.001$ & 25.0 & 37.6 & 0.001 \\
\hline Body mass index, $\mathrm{kg} / \mathrm{m}^{2}$ & & $24.3 \pm 2.7$ & $23.6 \pm 3.2$ & $<0.001$ & $24.3 \pm 2.7$ & $23.5 \pm 3.2$ & 0.001 \\
\hline Fasting blood glucose, mg/dL & & $99.0 \pm 25.3$ & $91.0 \pm 19.0$ & $<0.001$ & $98.2 \pm 27.8$ & $90.1 \pm 17.9$ & $<0.001$ \\
\hline Total cholesterol, mg/dL & & $197.7 \pm 37.1$ & $189.0 \pm 33.8$ & 0.002 & $195.6 \pm 36.9$ & $189.3 \pm 41.8$ & 0.037 \\
\hline Systolic blood pressure, $\mathrm{mmHg}$ & & $123.4 \pm 16.3$ & $117.9 \pm 14.4$ & $<0.001$ & $121.8 \pm 14.7$ & $117.6 \pm 14.3$ & 0.037 \\
\hline \multirow[t]{2}{*}{ Smoking status, \% } & Ex & 31.0 & 17.7 & $<0.001$ & 29.6 & 15.8 & $<0.001$ \\
\hline & Current & 29.9 & 29.2 & & 30.7 & 30.2 & \\
\hline Alcohol drinking (yes), \% & & 73.6 & 74.0 & 0.881 & 74.4 & 76.7 & 0.496 \\
\hline Exercise (yes), \% & & 63.2 & 59.6 & 0.228 & 62.5 & 61.8 & 0.850 \\
\hline Family history of CRC (yes), \% & & 5.0 & 2.3 & 0.005 & 5.1 & 2.0 & 0.011 \\
\hline
\end{tabular}

Values are mean \pm standard deviation (SD) for continuous data.

Body mass index $(\mathrm{BMI})=$ weight in kilograms divided by height in meters squared.

CRC: Colorectal cancer, KCPS-II: The Korean Cancer Prevention Study-II. 
Table 2 Hazard ratios for risk factors on risk of CRC: The Korean Cancer Prevention Study-II and the KCPS-II sub-cohort

\begin{tabular}{|c|c|c|c|}
\hline & & KCPS-II cohort (Whole participants) & KCPS-II sub-cohort (Case-cohort design) \\
\hline \multicolumn{2}{|l|}{ Traditional risk factors } & $\mathrm{HR}(95 \% \mathrm{Cl})$ & HR $(95 \% \mathrm{Cl})$ \\
\hline \multicolumn{2}{|l|}{ Age, year } & $1.07(1.06-1.08)$ & $1.08(1.07-1.10)$ \\
\hline \multicolumn{2}{|l|}{ Sex (female) } & $0.65(0.45-0.92)$ & $0.71(0.50-1.11)$ \\
\hline \multicolumn{2}{|l|}{ Log (fasting serum glucose), mg/dL } & $1.81(0.99-3.30)$ & $2.16(0.96-4.83)$ \\
\hline \multirow[t]{2}{*}{ Smoking status } & Ex-smoker & $1.45(1.03-2.05)$ & $1.74(1.12-2.72)$ \\
\hline & Current smoker & $1.28(0.90-1.83)$ & $1.40(0.91-2.17)$ \\
\hline \multicolumn{2}{|l|}{ Exercise (yes) } & $0.91(0.70-1.17)$ & $0.68(0.49-0.94)$ \\
\hline \multicolumn{2}{|l|}{ Family history of CRC (yes) } & $2.40(1.34-4.30)$ & $3.49(1.70-7.17)$ \\
\hline \multicolumn{2}{|l|}{ Per 1 SD of TRS increase } & $1.34(1.29-1.39)$ & $1.30(1.24-1.36)$ \\
\hline
\end{tabular}

CRC: colorectal cancer, HR: hazard ratios, $\mathrm{Cl}$ : confidence interval, SD: standard deviation, TRS: traditional risk score, TRS combined information on above 6 risk factors: age, sex, fasting serum glucose, exercise, and family history of CRC. KCPS-II: The Korean Cancer Prevention Study-II.

Table 3 Allelic odds ratios for subtype of CRC in the Korean Cancer Prevention Study II sub-cohort

\begin{tabular}{|c|c|c|c|c|c|c|c|}
\hline SNPs* & $\begin{array}{l}\text { Reference number in } \\
\text { Additional file 2: Table S1 }\end{array}$ & Chr. & RA & RAF & $\begin{array}{l}\text { Colorectal cancer } \\
\text { OR }(95 \% \mathrm{Cl})\end{array}$ & $\begin{array}{l}\text { Colon cancer } \\
\text { OR }(95 \% \mathrm{Cl})\end{array}$ & $\begin{array}{l}\text { Rectal cancer } \\
\text { OR }(95 \% \mathrm{Cl})\end{array}$ \\
\hline rs3802842 & $4,7,19,29,32,34,36$ & 11 & C & 0.40 & $1.46(1.14-1.86)$ & $1.30(0.93-1.81)$ & $1.50(1.10-2.04)$ \\
\hline rs4444235 & $4,7,32,38$ & 14 & C & 0.52 & $1.02(0.80-1.29)$ & $1.01(0.73-1.40)$ & $1.03(0.76-1.40)$ \\
\hline rs4939827 & $7,10,29,30,34,41,42,43,44,45,46$ & 18 & T & 0.22 & $1.32(1.01-1.71)$ & $1.04(0.71-1.52)$ & $1.55(1.11-2.16)$ \\
\hline rs6983267 & $7,16,17,18,19,20,21,22,23,24,25,26,27,28$ & 8 & G & 0.43 & $1.14(0.91-1.43)$ & $0.85(0.61-1.17)$ & $1.46(1.08-1.97)$ \\
\hline rs10505477 & $11,12,13,14,15$ & 8 & G & 0.43 & $1.15(0.92-1.45)$ & $0.88(0.64-1.21)$ & $1.44(1.06-1.94)$ \\
\hline rs10795668 & $7,20,30,31,32,33,34$ & 10 & G & 0.64 & $1.20(0.92-1.55)$ & $0.93(0.66-1.32)$ & $1.45(1.03-2.05)$ \\
\hline rs11169552 & 1 & 12 & $\mathrm{~T}$ & 0.34 & $0.98(0.76-1.25)$ & $1.05(0.75-1.47)$ & $0.93(0.67-1.28)$ \\
\hline rs6687758 & 1,2 & 1 & G & 0.29 & $0.96(0.74-1.25)$ & $1.14(0.80-1.63)$ & $0.85(0.60-1.20)$ \\
\hline rs7014346 & 29 & 8 & G & 0.69 & $0.94(0.73-1.21)$ & $1.08(0.76-1.54)$ & $0.86(0.62-1.18)$ \\
\hline rs11903757 & 3 & 2 & $\mathrm{~T}$ & 0.96 & $0.79(0.45-1.42)$ & $0.75(0.35-1.63)$ & $0.91(0.42-1.96)$ \\
\hline rs3217810 & 3 & 12 & C & 0.95 & $0.98(0.19-5.21)$ & $0.44(0.08-2.45)$ & $\mathrm{NE}$ \\
\hline rs10411210 & 20,28 & 19 & $\mathrm{~T}$ & 0.18 & $0.91(0.66-1.25)$ & $0.82(0.52-1.30)$ & $1.00(0.67-1.50)$ \\
\hline rs961253 & $4,7,11,19,20,34,38,47$ & 20 & A & 0.10 & $1.38(0.97-1.97)$ & $1.19(0.72-1.98)$ & $1.45(0.93-2.26)$ \\
\hline rs6691170 & $1,2,4$ & 1 & $\mathrm{~T}$ & 0.09 & $\mathrm{NE}$ & & \\
\hline rs9929218 & $20,21,31,38,40$ & 16 & A & 0.15 & $1.21(0.87-1.68)$ & $1.16(0.74-1.83)$ & $1.20(0.78-1.82)$ \\
\hline rs10911251 & 3 & 1 & C & 0.46 & $1.01(0.80-1.29)$ & $0.80(0.58-1.12)$ & $1.22(0.90-1.66)$ \\
\hline rs7758229 & 10 & 6 & $\mathrm{~T}$ & 0.22 & $1.06(0.80-1.41)$ & $0.91(0.60-1.37)$ & $1.20(0.84-1.71)$ \\
\hline rs59336 & 3 & 12 & $\mathrm{~T}$ & 0.63 & $0.94(0.73-1.20)$ & $0.79(0.57-1.12)$ & $1.09(0.79-1.52)$ \\
\hline rs3217901 & 37 & 12 & G & 0.65 & $1.10(0.87-1.39)$ & $1.51(1.08-2.11)$ & $0.83(0.61-1.12)$ \\
\hline rs10936599 & $1,4,5,6,7,8$ & 3 & $\mathrm{~T}$ & 0.61 & 1.09 (0.87-1.38) & $1.07(0.77-1.48)$ & $1.11(0.81-1.50)$ \\
\hline rs647161 & 9 & 5 & C & 0.69 & $0.95(0.73-1.22)$ & $0.72(0.51-1.01)$ & $1.25(0.88-1.77)$ \\
\hline rs7136702 & 1 & 12 & $\mathrm{~T}$ & 0.53 & $1.08(0.85-1.37)$ & $1.10(0.79-1.54)$ & $1.02(0.75-1.39)$ \\
\hline rs4779584 & $4,19,20,30,32,33,36,39$ & 15 & $\mathrm{~T}$ & 0.84 & $0.97(0.70-1.34)$ & $0.91(0.58-1.43)$ & $1.02(0.67-1.55)$ \\
\hline
\end{tabular}

CRC: colorectal cancer, Chr.: chromosome, RA: risky allele, RAF: risky allele frequency, OR: odds ratio, Cl: confidence interval, NE: not estimated due to small number, SNP with ORs in bold were selected for genetic risk score calculations.

* List of references and detailed information were summarized in Additional file 2: Table S1. 
Table 4 Area under receiver operating characteristic curve by subtype of CRC: Korean Cancer Prevention Study II sub-cohort

\begin{tabular}{|c|c|c|c|c|c|c|c|}
\hline & & \multicolumn{2}{|c|}{ Colorectal cancer } & \multicolumn{2}{|l|}{ Colon cancer } & \multicolumn{2}{|l|}{ Rectal cancer } \\
\hline & & HR (95\% Cl) & HR $(95 \% \mathrm{Cl})^{*}$ & HR $(95 \% \mathrm{Cl})$ & HR $(95 \% \mathrm{Cl})^{*}$ & HR (95\% Cl) & HR $(95 \% \mathrm{Cl})^{*}$ \\
\hline \multirow[t]{4}{*}{ TRS } & Q1 & 1.00 & 1.00 & 1.00 & 1.00 & 1.00 & 1.00 \\
\hline & Q2 & $1.97(0.95-4.11)$ & $2.03(0.98-4.22)$ & $1.60(0.52-4.94)$ & $1.60(0.52-4.95)$ & $2.28(0.87-6.01)$ & $2.40(0.91-6.31)$ \\
\hline & Q3 & $2.57(1.29-5.14)$ & $2.62(1.31-5.24)$ & $2.19(0.76-6.32)$ & $2.16(0.75-6.24)$ & $2.88(1.15-7.24)$ & $3.02(1.20-7.59)$ \\
\hline & Q4 & $11.29(6.06-21.1)$ & $11.54(6.19-21.5)$ & $13.33(5.31-33.5)$ & $13.27(5.29-33.3)$ & $10.16(4.36-23.7)$ & $10.59(4.54-24.7)$ \\
\hline \multirow[t]{4}{*}{ GRS } & Q1 & & 1.00 & & 1.00 & & 1.00 \\
\hline & Q2 & & $1.42(0.77-2.61)$ & & $0.95(0.47-1.91)$ & & $3.95(0.93-16.84)$ \\
\hline & Q3 & & $1.68(0.92-3.07)$ & & $0.77(0.37-1.59)$ & & $7.06(1.70-29.28)$ \\
\hline & Q4 & & $2.65(1.43-4.91)$ & & $1.17(0.55-2.50)$ & & $10.83(2.58-45.40)$ \\
\hline AUROC & & $0.73(0.69-0.78)^{\dagger}$ & $0.74(0.70-0.78)$ & $0.76(0.70-0.83)^{\dagger}$ & $0.75(0.69-0.81)$ & $0.71(0.65-0.77)^{\dagger}$ & $0.74(0.68-0.79)$ \\
\hline NRI & & - & $0.17(-0.05-0.37)$ & & $-0.17(-0.33-0.21)$ & - & $0.41(0.10-0.68)$ \\
\hline$P$ for NRI & & & 0.108 & & 0.688 & & 0.008 \\
\hline
\end{tabular}

CRC: colorectal cancer, TRS: traditional risk score, GRS: genetic risk score, HR: hazard ratio, Cl: confidence interval, AUROC: Area under receiver operating characteristic curve, NRI: net reclassification index.

*Combined model, ${ }^{\dagger}$ AUROC for TRS alone, AUROC for TRS + GRS.

Recent genome-wide association studies (GWASs) have identified a number of common genetic markers significantly associated with CRC [6,11-13]. However, most of these GWAS results have been from populations of European descent. In any GWAS results, the risk associated with any one marker is individually modest, because these markers are rarely causal but merely tag regions haplotypes spanning chromosomal regions. Thus, predicted risks for individuals tend to be very modest and rarely exceed thresholds that would trigger any clinical intervention, and at best these predicted risk might be useful for identifying sub-groups of high-risk subjects carrying multiple risk alleles. Companies such as DeCODEme and 23andme include panels of common SNPs in their testing panels and report predicted risk for complex diseases such as $\mathrm{CRC}$, yet research suggests any prediction based on genetic markers identified through genome-wide studies is of questionable clinical utility [6].

\section{Present study findings}

During the follow-up period which included 633,210 person-year coverage, 258 incident CRC cases (196 men and 62 women) occurred. This case-cohort study evaluated the ability to predict risk based on TRS alone, and these plus a GRS which aggregates information from 7 genetic markers shown to be associated with risk of CRC in Koreans. While most genetic epidemiologic studies have focused on the combined outcome CRC (colon or rectal cancer), but showed less improvement for CRC and colon cancer alone in our Korean sub-cohort study. The rectal cancer prediction model using both TRS and GRS had an increased AUROC by about 3\% compared to the AUC from a TRS model (Table 4). The prediction model for rectal cancer alone showed a substantial increase in NRI of about $41 \%$.

We set out to develop and validate CRC risk prediction models and assess their performance in profiling

\section{Colorectal cancer}

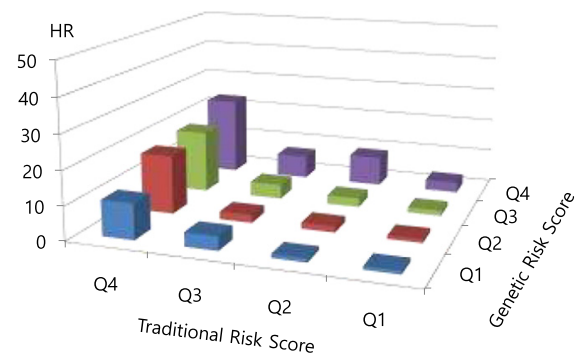

\section{Rectal cancer}

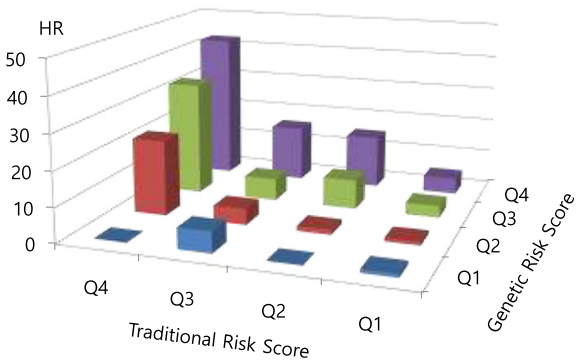

Figure 1 Combined effect of traditional risk score and genetic risk score on colorectal cancer: Korean Cancer Prevention Study-ll. 
individual genetic risk of CRC in Koreans. We developed models incorporating age, gender, fasting serum glucose, smoking, exercise, family history $(\mathrm{FH})$ and genotype data from 23 common genetic markers reported to significantly associate with CRC in over 47 previous publications. Several of these 23 SNPs (rs3802842, rs4939827, rs6983267, rs10795668, rs961263, rs4779584, and so on) have been well replicated in the scientific literature (Table 3). In Koreans, 7 SNPs (rs3802842, rs4939827, rs6983267, rs10505477, rs10795668, rs961263, rs9929218) among the 23 SNPs were associated with CRC in our sub-cohort based on 258 incident cases. However, some of these 7 SNPs showed positive association with wide $95 \%$ confidence intervals.

\section{CRC versus colon and rectal cancer}

Previous GWAS using CRC as the outcome (combining colon and rectal cancer together) reported genome-wide significant associations between risk and multiple SNPs [11-13]. But few studies have considered colon and rectal cancer separately. Some studies of environmental factor argue differences between CRC sub-types may be important [8-9].

When we separated our CRC cases into colon and rectal cancer groups, 7 out of 23 reported risk SNPs showed statistically significant association with CRC and rectal cancer, but not with colon cancer (Table 3). These SNPs showed consistent direction of association and effect size, and the lack of statistical significance could just reflect a loss of power due to smaller sample sizes.

This suggests future studies should also separate colon and rectal cancer rather than just testing only the combined outcome CRC. Also, it raises the question of whether separate prediction models for colon and rectal cancer should be developed.

\section{TRS versus $G R S$}

In this study of CRC alone, TRS alone showed a strong predictive power of 0.73 , and the addition of a GRS failed to show significant contribution or change. In the combined risk models, however, that including both the TRS and GRS, rectal cancer showed the greatest improvement $(\mathrm{ROC}$ area change $=3 \%$; NRI $=0.41)$.

Recently, Dunlop et al. (2013) [6] conducted a ROC analysis of models including genotype data alone or in combination age, gender and FH showed very modest discrimination across the full risk spectrum of risk, with $\mathrm{AUC}=0.59$ and 0.57 (internal validation) or 0.56 and 0.57 (external validation sets). Their overall positive predictive value fell between 0.51 and 0.71 .

The modest performance in individualized CRC risk profiling is consistent with risk prediction studies for other complex diseases (coronary heart disease [14], stroke $[15,16]$, and age-related macular degeneration [17]).
The best predictive performances have been obtained by combining genetic, demographic and environmental variables [17]. In our study, GRS itself showed similar ROC value ( 0.6). However, when we combined GRS with traditional risk factors (like age, sex, high fasting glucose, smoking, exercise, and family history) the ROC increased up to 0.74 for predicting $\mathrm{CRC}$, and similar models for rectal cancer showed greater increase.

\section{Limitation and strength}

Major limitations included reliance on self-reported exposures at a single point in time, thus precluding the definitive exclusion of potential misclassification. The statistical power of the current study is modest, as genotyping was performed on a limited sample size of CRC cases and controls. A strong point of our study is the case-cohort design drawn from an underlying large prospective cohort. Case identifications were performed by record linkage to the national cancer registry with verification.

\section{Conclusion}

In conclusion, findings in this current study provide some evidence of improved prediction for CRC in models combining traditional and genetic risk factors. This emphasizes both genetic and traditional factors associated with $\mathrm{CRC}$ should be considered when predicting risk.

\section{Methods}

\section{Study subjects}

We have used data on the Korean Metabolic Syndrome Research Initiative in Seoul, initiated in 2005. We have labeled this study as the Korean Cancer Prevention Study-II (KCPS-II). A full description of KCPS-II has been previously published $[9,18]$. Study members were recruited from participants in routine health assessments at health promotion centers in Seoul and GyeongGi province, South Korea, between 2004 and 2011. Twenty one centers holding electronic health records agreed to linkage of participants' records to national cancer registry for monitoring of cancer events. The initial study population included 190,332 individuals (112,852 men, 77,480 women), aged $20-94$ years. About $90 \%$ of participants were enrolled between 2005 and 2008, and the remaining were enrolled prior to or after this period. We have acquired both written consent forms and blood samples from 157,526 participants. Among the total 157,526 participants, 174 participants who reported of having prevalent CRC were excluded. In addition, 11,510 participants who had missing values on body mass index, fasting blood glucose, total cholesterol, systolic blood pressure, smoking status, alcohol drinking, and exercise were excluded. Follow up of participants through December 2011, identified 258 out of these 145,842 participants as incident cases of colorectal cancer. 
For the case-cohort study, we selected a sub-cohort as a $1 \%$ random sample of all participants. Two of 1,514 randomly selected participants were found to be diagnosed with CRC from our sub-cohort study, while 173 CRC cases were verified outside the sub-cohort. In short, a total of 1,685 additional participants (1,514 plus 173 participants minus 2 participants) were included in our case-cohort study design. Until 2012, the actual number of CRC patients eligible for genetic testing was 173 among all known CRC cases 258. The remaining 85 CRC patients will be tested during the next phase of our study. The Institutional Review Board of Yonsei University reviewed and approved this study.

\section{Traditional risk score}

To develop the traditional risk score (TRS), Cox proportional hazards regression models were fitted first to a basic set of classical risk factors: age, sex, smoking status, fasting serum glucose, family history of colorectal cancer. The TRS algorithm is given in online Additional file 1.

\section{SNP genotyping}

Twenty-three single-nucleotide polymorphisms (rs3802842, rs4444235, rs4939827, rs6983267GG, rs10505477, rs10795668, rs11169552, rs6687758, rs7014346, rs11903757, rs3217810, rs10411210, rs961253, rs6691170, rs9929218, rs10911251, rs7758229, rs59336, rs3217901, rs10936599, rs647161, rs7136702TT, rs4779584) identified in previous 47 studies were genotyped (Table 3 and Additional file 2: Table S1). DNA was isolated from peripheral blood of participants and genotyped at DNA Link Inc. (Seoul, Korea). The genotyping was performed using SNP type assay (Fluidigm, San Francisco, CA, USA) following the manufacturer's recommendation. Genomic DNA flanking these SNPs of interest was amplified with PCR reaction with STA primer set and Qiagen 2X Mutiplex PCR Master Mix (Qiagen) in 5 microliter reaction volume, containing $60 \mathrm{ng}$ of genomic DNA. PCR reactions were carried out as follows: $15 \mathrm{~min}$ at $95^{\circ} \mathrm{C}$ for 1 cycle, and 14 cycles on $95^{\circ} \mathrm{C}$ for $15 \mathrm{~s}$ and $60^{\circ} \mathrm{C}$ for $4 \mathrm{~min}$. After amplification, the the STA products were diluted 1:100 in DNA Suspension Buffer. A 2.5 microliter of the diluted STA products were added to a Sample Pre-Mix containing 3 microliter of 2X Fast Probe Master Mix, 0.3 microliter of the SNP type 20X Sample Loading Reagent, 0.1 microliter of the SNP type Reagent, and 0.036 microliter of the ROX. After the Assay Pre-Mix and the Sample Pre-Mix were loaded into the 48.48 Dynamic Array, SNP type assay reaction was carried out. Analysis was carried out using Fluidigm SNP Genotyping Analysis software (version 4.0.1; Fluidigm). Internal quality control (QC) measures were employed to ensure accuracy of the data. A total of 1,685 individuals were genotyped on this platform.

\section{Anthropometric measurements}

Each participant was interviewed using a structured questionnaire to collect information on smoking status and alcohol consumption as well as demographic characteristics, such as age, gender, and family history of various diseases. Cigarette smoking was classified into never smokers, exsmokers, and current smokers. Alcohol consumption was divided into nondrinkers and current drinkers. Regular physical activity was tracked as either "yes" or "no". Participant height and weight were measured while the participants were wearing light clothing. Body mass index (BMI) was calculated by dividing the weight $(\mathrm{kg})$ by the square height $\left(\mathrm{m}^{2}\right)$. Systolic and diastolic blood pressures were measured after a rest period of at least $15 \mathrm{~min}$.

\section{SNP selection and GRS calculation}

Each SNP in this study was assumed to be associated with risk following an additive genetic model, which is considered to be generally robust even when the true genetic model is not known or may be incorrectly specified [19]. The GRS was created by two methods: a simple count method (count GRS) and a weighted method (weighted GRS) [14,20]. Both methods assumed each SNP to be independently associated with the risk of CRC (i.e. no interaction). We assumed an additive genetic model for each SNP, applying a linear weighting of 0 , 1 , or 2 to genotypes containing 0,1 , or 2 of the reported risk alleles, respectively. This count model assumes each SNP in the panel contributes equally to the risk for CRC and was calculated by summing the values for each SNP. The weighted GRS was calculated by multiplying each estimated beta-coefficient by the number of corresponding risk alleles $(0,1$, or 2$)$.

In this study, traditional risk factor score (TRS) combined information on 6 risk factors: age, sex, fasting serum glucose, smoking status, exercise status, and family history of CRC.

\section{Outcome classification}

The principle outcome variable was incidence of CRC ( $\mathrm{n}=258$ in whole participants, $\mathrm{n}=173$ in the sub-cohort), based on data from the national cancer registry. According to the International Classification of Diseases, Tenth Revision (ICD-10), CRC was coded as C18-C20 (C18 for colon, C19 for rectosigmoid, and C20 rectum) [21].

\section{Statistical analysis}

All statistical tests were two-sided, and statistical significance was determined as $\mathrm{p}<0.05$. To evaluate general characteristics of the study population, means and standard deviations (SD) were calculated, and frequencies of cigarette smoking, alcohol consumption, and physical activity was determined. A $\chi^{2}$ goodness-of-fit test was used to assess whether SNPs were in Hardy-Weinberg 
Equilibrium and to determine differences in genotype frequencies between CRC cases and controls. The GRS was categorized into quartiles. CRC risk associated with any one genotype was estimated as OR and 95\% confidence interval (CI), and was computed using logistic regression under an additive genetic model. We also used receiver operating characteristic (ROC) curve analysis and calculated the area under the curve (AUC; also known as the C statistic) and the continuous net reclassification index (NRI) to evaluate the discrimination power of a CRC risk model. Finally, Cox proportional hazards models were used to estimate the effect of GRS and TRS on CRC risk in our case-cohort design.

\section{Availability of supporting data}

The data set supporting the results of this article is available in the LabArchives, in https://mynotebook.labarchives.com/.

\section{Additional files}

Additional file 1: The traditional risk score (TRS).

Additional file 2: Table S1. Colorectal cancer related 47 references selected for the present study.

\section{Abbreviations}

GRS: Genetic risk score; TRS: Traditional risk score; CRC: Colorectal cancer; OR: Odd ratio; Cl: Confidence interval; SNP: Single nucleotide polymorphisms.

\section{Competing interests}

All authors declare that they have no competing interests.

\section{Authors' contributions}

KJJ and SK: data analysis, and writing the manuscript; DW, CJ, and TK: writing the manuscript, SHJ: study design, collecting data, and data analysis, THB: writing the manuscript. All author read and approved the final manuscript.

\section{Acknowledgments}

This work was supported by a grant from the National R\&D Program for Cancer Control; Ministry for Health, Welfare and Family Affairs, Republic of Korea (1220180)

\section{Author details \\ 'Department of Public Health, Graduate School, Yonsei University, Seoul, South Korea. ${ }^{2}$ The Catholic University of Korea, Seoul Saint Mary's Hospital, Seoul, South Korea. ${ }^{3}$ Institute for Health Promotion and Department of Epidemiology and Health Promotion, Graduate School of Public Health, Yonsei University, 50 Yonse-ro, Seodaemun-gu, Seoul, South Korea. ${ }^{\text {DDivision }}$ of Gastroenterology, Department of Internal Medicine, Yonsei University College of Medicine, Seoul, South Korea. ${ }^{5}$ Johns Hopkins Bloomberg School of Public Health, Baltimore, MD, USA.}

Received: 7 January 2015 Accepted: 22 April 2015

Published online: 09 May 2015

\section{References}

1. National Cancer Information Center. Cancer Incidence trend analysis. accessed online Date on June 2014 at http://www.cancer.go.kr/mbs/cancer/ subview.jsp?id=cancer_040104000000

2. Yarnall JM, Crouch DJM, Lewis CM. Incorporating non-genetic risk factors and behavioural modifications into risk prediction models for colorectal cancer. Cancer Epidemiol. 2013;37(3):324-9.

3. The International Schizophrenia Consortium. Common polygenic variation contributes to risk of schizophrenia and bipolar disorder. Nature. 2009;460(7256):748-52
4. Jiang $H$, Liu F, Wang Z, Na R, Zhang L, Wu Y, et al. Prediction of prostate cancer from prostate biopsy in Chinese men using a genetic score derived from 24 prostate cancer risk-associated SNPs. Prostate. 2013;73(15):1651-9.

5. Blakely T, Barendregt JJ, Foster RH, Hill S, Atkinson J, Sarfati D, et al. The association of active smoking with multiple cancers: national census-cancer registry cohorts with quantitative bias analysis. Cancer Causes Control. 2013;24(6):1243-55.

6. Dunlop MG, Tenesa A, Farrington SM, Ballereau S, Brewster DH, Koessler T, et al. Cumulativeimpact of commongeneticvariants and otherriskfactors on colorectalcancerrisk in 42,103 individuals. Gut. 2013;62(6):871-81.

7. Shin A, Joo J, Yang HR, Bak J, Park Y, Kim J, et al. Risk prediction model for colorectal cancer: National Health Insurance Corporation study, Korea. PLoS One. 2014;9(2):e88079.

8. Shin A, Joo J, Bak J, Yang HR, Kim J, Park S, et al. Site-specific risk factors for colorectal cancer in a Korean population. PLoS One. 2011:6(8):e23196.

9. Shin HY, Jung KJ, Linton JA, Jee SH. Association between fasting glucose levels and incidence of colorectal cancer in Korean men: the Korean cancer prevention study-II. Metabolism 2014 Jul 10. [Epub ahead of print].

10. Lichtenstein P, Holm NV, Verkasalo PK, Iliadou A, Kaprio J, Koskenvuo M, et al. Environmental and heritable factors in the causation of cancer-analyses of cohorts of twins from Sweden, Denmark, and Finland. N Engl J Med. 2000;13(343(2)):78-85

11. Tomlinson IP, Webb E, Carvajal-Carmona L, Broderick P, Howarth K, Pittman AM, et al. A genome-wide association study identifies colorectal cancer susceptibility loci on chromosomes 10p14 and 8q23.3. Nat Genet. 2008;40(5):623-30. doi:10.1038/ng.111. Epub 2008 Mar 30.

12. Tenesa A, Farrington SM, Prendergast JG, Porteous ME, Walker M, Haq N, et al. Genome-wide association scan identifies a colorectal cancer susceptibility locus on 11q23 and replicates risk loci at 8q24 and 18q21. Nat Genet. 2008;40(5):631-7. doi:10.1038/ng.133. Epub 2008 Mar 30.

13. Spain SL, Carvajal-Carmona LG, Howarth KM, Jones AM, Su Z, Cazier JB, et al. Refinement of the associations between risk of colorectal cancer and polymorphisms on chromosomes $1 \mathrm{q} 41$ and 12q13.13. Hum Mol Genet. 2012;21(4):934-46. doi:10.1093/hmg/ddr523. Epub 2011 Nov 10.

14. Ripatti S, Tikkanen E, Orho-Melander M, Havulinna AS, Silander K, Sharma A, et al. A multilocus genetic risk score for coronary heart disease: case-control and prospective cohort analyses. Lancet. 2010:376(9750):1393-400.

15. Ibrahim-Verbaas CA, Fornage M, Bis JC, Choi SH, Psaty BM, Meigs JB, et al. Predicting stroke through genetic risk functions: the CHARGE Risk Score Project. Stroke. 2014;45(2):403-12. Epub 2014 Jan 16.

16. Malik R, Bevan S, Nalls MA, Holliday EG, Devan WJ, Cheng YC, et al Wellcome Trust Case Control Consortium 2. Multilocus genetic risk score associates with ischemic stroke in case-control and prospective cohort studies. Stroke. 2014;45(2):394-402. Epub 2014 Jan 16.

17. Seddon JM, Reynolds R, Maller J, Fagerness JA, Daly MJ, Rosner B. Prediction model for prevalence and incidence of advanced age-related macular degeneration based on genetic, demographic, and environmental variables. Invest Ophthalmol Vis Sci. 2009:50(5):2044-53. doi:10.1167/iovs.08-3064. Epub 2008 Dec 30

18. Jo J, Nam CM, Sull JW, Yun JE, Kim SY, Lee SJ, et al. Prediction of Colorecta Cancer Risk Using a Genetic Risk Score: The Korean Cancer Prevention Study-II (KCPS-II). Genomics Inform. 2012;10(3):175-83.

19. Balding DJ. A tutorial on statistical methods for population association studies. Nat Rev Genet. 2006;7(10):781-91.

20. Cornelis MC, Qi L, Zhang C, Kraft P, Manson J, Cai T, et al. Joint effects of common genetic variants on the risk for type 2 diabetes in U.S. men and women of European ancestry. Ann Intern Med. 2009;150(8):541-50.

21. World Health Organization. International Statistical Classification of Diseases and Related Health Problems. 10th Rev. Geneva: World Health Organization; 1992. 